\title{
Sampled Tracking for Delayed Systems Using Two- Time-Scale Sampled-data Controllers
}

\author{
Haoping Wang ${ }^{1}$, Christian Vasseur ${ }^{2}$, Vladan Koncar ${ }^{3}$, Afzal Chamroo ${ }^{4}$, Nicolai Christov ${ }^{5}$ \\ 1,2,5 LAGIS CNRS FRE 3303, Université Lille 1 Sciences et Technologies, Bât. P2, \\ 59655 Villeneuve d'Ascq, France, \\ \{haoping.wang, christian.vasseur, nicolai.christov\}@univ-lille1.fr \\ ${ }^{3}$ GEMTEX, ENSAIT, \\ 9 Rue de l'Ermitage, BP 30329, 59056 Roubaix, France, \\ vladan.koncar@ensait.fr \\ ${ }^{4}$ LAII, Université Poitiers, \\ 40 av. du Recteur Pineau, 86022 Poitiers, France, \\ afzal.chamroo@univ-poitiers.fr
}

\begin{abstract}
This article deals with the trajectory tracking of linear plants with sampled and delayed outputs. A class of sampled-data controllers with two time-scales is proposed which allows sampled tracking with a delay twice as much as that of the plant output. Numerical simulation results are presented to demonstrate the effectiveness of the proposed approach.
\end{abstract}

Keywords: Sampled tracking, sampled-data controllers, two time-scale systems, optimal control, Luenberger discrete-time observer.

\section{Introduction}

During the last two decades, two-time-scale sampled-data control of continuous-time plants has been studied in several papers dealing with both its theory and applications [1]-[5]. For instance, a sampled-data control scheme which control actions are scheduled at two different sampling rates (slow and fast) is suggested in [1]. This sampled-data control is in composite form and is computed as the sum of the slow and fast control signals. A two-time-scale digital controller is derived by using the singular perturbation theory and is applied for motor position servoing [2]. Recently, a robust two-time-scale control based on the singular perturbation method and time delay control is proposed for a pneumatic vibration isolator [4]. A decentralised two-time-scale motions control of linear time-invariant plants with unstable decentralised fixed modes (UDFM) is designed in [5]. The method used generalizes the sampled-data hold function introduced by Kabamba [6] to eliminate UDFM and to decouple the discrete-time plant model into independent input-output channels.

An alternative approach for two-time-scale controller design is proposed in [3] using the theory of the sampled-data systems with piecewise functioning (SPF) [3]. The developed controller, however, needs full information for the plant state which limits its practical application.

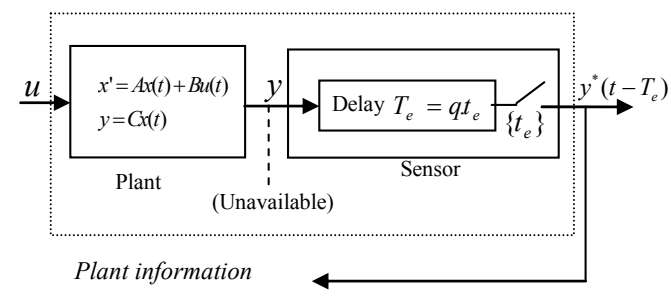

Figure 1. System to be controlled

In this paper we deal with the case when the only available plant information is delivered from the plant output via a digital sensor introducing a delay $T_{e}$ corresponding to the time needed to process the information (see Figure 1). Further on we consider that $T_{e}=q t_{e}$, where $t_{e}$ is the sampling period. Such systems are frequently encountered in industry when digital technology is used for measurement (e.g. camera) and/or control [8]. Their dynamics can be described as

$$
\begin{aligned}
& \dot{x}(t)=A x(t)+B u(t) \\
& y=C x(t) \\
& y^{*}\left(t-T_{e}\right)=y^{*}\left(t-q \cdot t_{e}\right)
\end{aligned}
$$

where $A \in \mathfrak{R}^{n \times n}, \quad B \in \mathfrak{R}^{n \times r} \quad$ and $C \in \mathfrak{R}^{m \times n}$ are constant matrices and * represents sampling operation with constant period $t_{e}$.

To ensure efficient tracking control of the considered systems, we propose a new type 
of SPF based two-time-scale sampled-data controllers (SDC) which use sampled and delayed plant output measurements.

The paper is organized as follows. In Section 2 the SPF theory and the existing full state information SDC are briefly presented. In Section 3 we develop SDC which use delayed state measurements and in Section 4 we generalize these controllers for the case when only delayed plant output measurements are available. The performance analysis of the new class of SDC by numerical simulation is presented in Section 5. Finally, in Section 6 some concluding remarks are given.

\section{Sampled-data Systems and Controllers with Piecewise Functioning}

The basic concept in SPF theory is the twotime scale illustrated in Figure 2 [3].

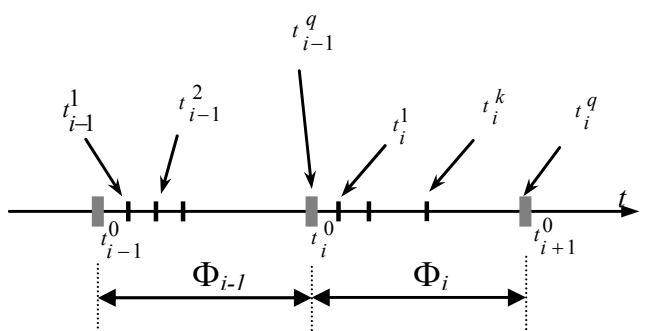

Figure 2. Sampled-data two-time scale

The discrete instants $t_{i}^{k}=i T_{e}+k t_{e} \quad$ are characterized by the indices:

$i$ - indicating the time scale relative to switching instants $i T_{e}, i=0,1,2, \ldots$, and

$k$-specifying the time scale relative to the system evolution between two switching instants.

Two successive switching instants $t_{i}^{0}$ and $t_{i+1}^{0}$ delimit an interval noted $\Phi_{i}$. In $\Phi_{i}$ the index $i$ does not change while $k$ increases from 0 to $q$. One has $t_{i-1}^{q}=t_{i}^{0}$.

A linear sampled-data system with piecewise functioning is a system whose dynamics can be described over all intervals $\Phi_{i}$ as

$$
\begin{aligned}
& x_{i}^{0}=B_{s} v_{i}^{0} \\
& x_{i}^{k+1}=A_{d} x_{i}^{k}+B_{d} u_{i}^{k}, k=0,1, \ldots, q-1 \\
& y_{i}^{k}=C x_{i}^{k}
\end{aligned}
$$

where $x_{i}^{k} \in \mathfrak{R}^{n}$ is the state vector, $u_{i}^{k} \in \mathfrak{R}^{r}$ is the control vector in $\Phi_{i}, \quad v_{i}^{0} \in \mathfrak{R}^{s}$ is the control vector at the switching instant $t_{i}^{0}$ and $y_{i}^{k} \in \mathfrak{R}^{m}$ is the output vector.

Denote the time $t$ in the interval $\Phi_{i}$ as $\tau+i T_{e}$, where $\tau \in\left[0, T_{e}\right]$. Thus we have

$x(t)=x\left(i T_{e}+\tau\right)=x_{i}(\tau)$

$u(t)=u_{i}(\tau)$

and in $\Phi_{i}$ the system (1) can be written as

$$
\begin{aligned}
& \dot{x}_{i}(\tau)=A x_{i}(\tau)+B u_{i}(\tau) \\
& y_{i}(\tau)=C x_{i}(\tau) \\
& y^{*}\left(t-T_{e}\right)=y_{i-1}(\tau)=C x_{i-1}(\tau) .
\end{aligned}
$$

Discretizing (5) we obtain the SPF

$$
\begin{aligned}
& x_{i}^{k+1}=A_{d} x_{i}^{k}+B_{d} u_{i}^{k}, k=0,1, \ldots, q-1 \\
& y_{i}^{k}=C x_{i}^{k} \\
& y_{i-1}^{k}=C x_{i-1}^{k}
\end{aligned}
$$

with

$A_{d}=e^{A t_{e}}, B_{d}=\int_{0}^{t_{e}} e^{A \cdot\left(t_{e}-\tau\right)} B d \tau$ and $v_{i}^{k}=x_{i}^{k}$.

Based on the SPF theory, a two-time-scale sampled-data controller (SDC) has been developed for plants with full state information realizing state trajectory tracking with one switching period of delay [3]. On the interval $\Phi_{i}$, the SDC is defined as

$$
\begin{aligned}
& \lambda_{i}^{0}=\beta_{s} \psi_{i}^{0} \\
& \lambda_{i}^{k+1}=\alpha \lambda_{i}^{k}+\beta a_{i}^{k}, k=0,1, \ldots, q-1 \\
& u_{i}^{k}=\gamma \lambda_{i}^{k+1}
\end{aligned}
$$

where $\quad \lambda_{i}^{k} \in \mathfrak{R}^{n} \quad$ and $\quad u_{i}^{k} \in \mathfrak{R}^{r} \quad$ denote respectively the SDC state and the output at the instant $t_{i}^{k}, \quad \psi_{i}^{0} \in \mathfrak{R}^{\sigma}$ is the input at switching instants, and $\alpha \in \mathfrak{R}^{n \times n}, \quad \beta \in \mathfrak{R}^{n \times r}$, $\beta_{s} \in \mathfrak{R}^{n \times \sigma}$ and $\gamma \in \mathfrak{R}^{r \times n}$ are constant matrices with appropriate dimensions. The matrix $\alpha$ is chosen so as to ensure the stability of SDC. For simplified SDC $\beta$ is a zero matrix and $\gamma$ is an arbitrary matrix. The SDC representation is given in Figure $3 \mathrm{a}$ and Figure $3 \mathrm{~b}$. Generally, $\lambda_{i}^{0} \neq \lambda_{i-1}^{q}$ which implies discontinuity at switching instants, as illustrated in Figure 3c. 


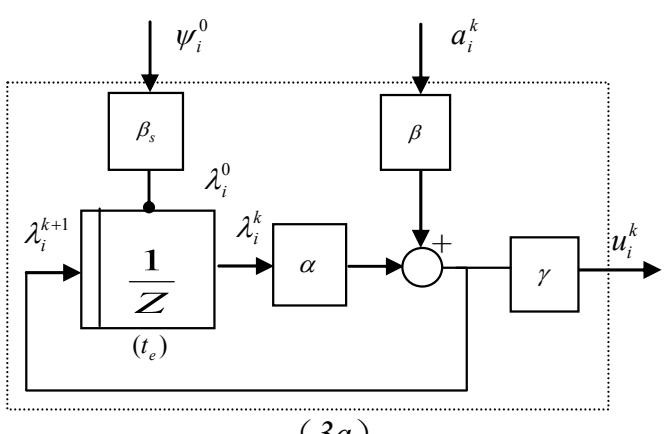

$(3 a)$

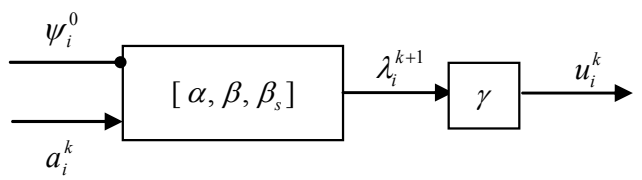

$(3 b)$

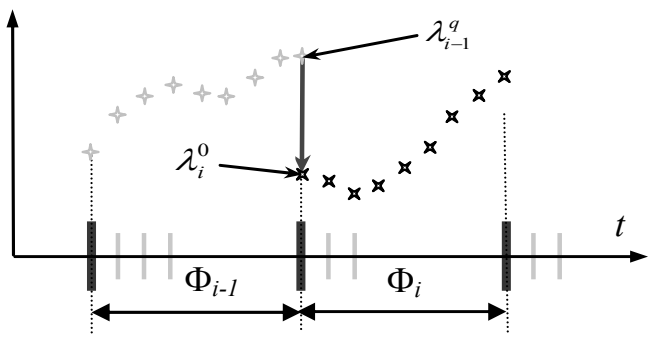

(3c)

Figure 3. Two-time-scale SDC

a. Detailed representation

b. Symbolic representation

c. Evolution of the controller state

Denote by $c(t)$ the desired plant state trajectory. The tuning of simplified SDC consists of determining $\psi_{i}^{0}$ and $\beta_{s}$ in order to ensure sampled trajectory tracking with one switching period of delay:

$x_{i+1}^{0}=c_{i}^{0} \quad \forall i=0,1,2, \ldots$

From (6) and (7) one obtains

$x_{i}^{q}=A_{d}^{q} x_{i}^{0}+M \lambda_{i}^{0}$

with

$M=\left[A_{d}^{q-1} B_{d}, A_{d}^{q-2} B_{d}, \ldots, A_{d}^{0} B_{d}\right]\left[\begin{array}{c}\gamma \alpha^{0} \\ \gamma \alpha^{1} \\ \vdots \\ \gamma \alpha^{q-1}\end{array}\right]$.

Taking into account (8) one has

$\beta_{s} \psi_{i}^{0}=M^{-1}\left[c_{i}^{0}-A_{d}^{q} x_{i}^{0}\right]$

and thus

$\beta_{s}=M^{-1}, \psi_{i}^{0}=c_{i}^{0}-A_{d}^{q} x_{i}^{0}$.
The SDC performances can be improved minimizing the oscillation effects as shown in [11], [3]. Define the cost function

$J=\frac{1}{2} \sum_{k=0}^{q-1}\left[\left(c_{i-1}^{k}-x_{i}^{k}\right)^{T} Q\left(c_{i-1}^{k}-x_{i}^{k}\right)+u_{i}^{k T} R u_{i}^{k}\right]$

where $Q \in \mathfrak{R}^{n \times n}$ and $R \in \mathfrak{R}^{r \times r}$ are positive definite matrices. The minimization of $J$ ensures the reduction of intersample ripples and the moderation of control magnitude. Denote the Hamiltonian of the optimal control problem (6a), (13) by

$$
\begin{aligned}
H= & -\frac{1}{2} \sum_{k=0}^{q-1}\left[\left(c_{i-1}^{k}-x_{i}^{k}\right)^{T} Q\left(c_{i-1}^{k}-x_{i}^{k}\right)+u_{i}^{k T} R u_{i}^{k}\right] \\
& +\sum_{k=0}^{q-1} l_{i}^{(k+1) T}\left[A_{d} x_{i}^{k}+B_{d} u_{i}^{k}\right]
\end{aligned}
$$

where $l_{i}^{k} \in \mathfrak{R}^{n}$ is the Lagrange multiplier. Using the Pontryagin maximum principle one obtains the optimal control law

$$
\begin{aligned}
& l_{i}^{k+1}=A_{d}^{-T} l_{i}^{k}-A_{d}^{-T} Q\left(c_{i-1}^{k}-x_{i}^{k}\right) \\
& u_{i}^{k}=R^{-1} B_{d}^{T} l_{i}^{k+1}
\end{aligned}
$$

which can be realized by a SDC (7) with

$\lambda_{i}^{k}=l_{i}^{k}, \alpha=A_{d}^{-T}, \beta=A_{d}^{-T} Q, \gamma=R^{-1} A_{d}^{-T}$.

In this case

$$
\begin{aligned}
& \beta_{s}=\Theta_{12}^{-1} \\
& \psi_{i}^{0}=\left[c_{i}^{0}-\Theta_{11} x_{i}^{0}-I_{h}\right]
\end{aligned}
$$

where

$$
\begin{aligned}
& {\left[\begin{array}{ll}
\Theta_{11} & \Theta_{12} \\
\Theta_{21} & \Theta_{22}
\end{array}\right]=} \\
& {\left[\begin{array}{cc}
A_{d}-B_{d} R^{-1} B_{d}^{T} A_{d}^{-T} Q & B_{d} R^{-1} B_{d}^{T} A_{d}^{-T} \\
-A_{d}^{-T} Q & -A_{d}^{-T}
\end{array}\right]^{q}}
\end{aligned}
$$

$\left[\begin{array}{c}I_{h} \\ I_{b}\end{array}\right]=\left[\begin{array}{lllll}H^{q-1} K & H^{q-2} K \ldots H^{0} K\end{array}\right]\left[\begin{array}{c}c_{i-1}^{0} \\ c_{i-1}^{1} \\ \vdots \\ c_{i-1}^{q-1}\end{array}\right]$

and

$$
\begin{aligned}
H & =\left[\begin{array}{cc}
A_{d}+B_{d} R^{-1} B_{d}^{T} A_{d}^{-T} Q & B_{d} R^{-1} B_{d}^{T} A_{d}^{-T} \\
A_{d}^{-T} Q & A_{d}^{-T}
\end{array}\right] \\
K & =\left[\begin{array}{c}
-B_{d} R^{-1} B_{d}^{T} A_{d}^{-T} Q \\
-A_{d}^{-T} Q
\end{array}\right] .
\end{aligned}
$$


In the next two sections we shall generalize the presented full state information SDC for the cases of delayed state and output plant information.

\section{Sampled Tracking with Delayed State Feedback}

In this section, we assume that for system (6) delayed state measurements $x_{i-1}^{k}$ are only available. To solve the sampled tracking problem in this case we propose a new class of SDC incorporating an estimator of $x_{i}^{k}$ based on the measurements $x_{i-1}^{k}$ and the control signals $u_{i}^{k}$.

Using the system equation (6a) the state vector $x_{i}^{k}$ can be estimated as

$$
x_{i}^{k}=A_{d}^{q} x_{i-1}^{k}+\left[\begin{array}{lll}
A_{d}^{q-1} B_{d} & A_{d}^{q-2} B_{d} \ldots A_{d}^{0} B_{d}
\end{array}\right]\left[\begin{array}{c}
u_{i}^{k-q} \\
u_{i}^{k-q+1} \\
\vdots \\
u_{i}^{k-1}
\end{array}\right]
$$

Thus the sampled tracking with delayed state information can be realized by a controller consisting of a serial connexion of the estimator (24) and the standard full state information SDC (7).

When simplified full state information SDC is used, the controller parameters are determined from (12) and the corresponding controller structure is represented in Figure 4.

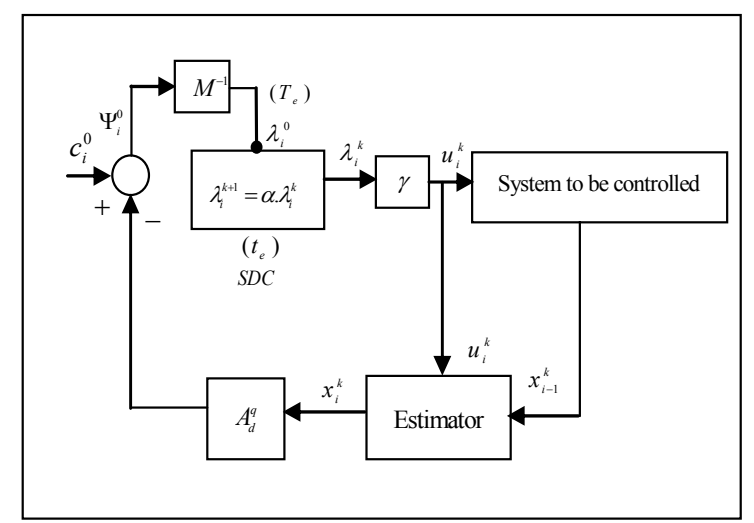

Figure 4. Simplified SDC with delayed state information

The controller performances can be optimized using the optimal full state information SDC presented in Section 2. In this case the controller parameters are determined from (17)-(24). The optimal controller structure is represented in Figure 5, where $\Sigma(\cdot)$ is defined by equations (21)-(23).

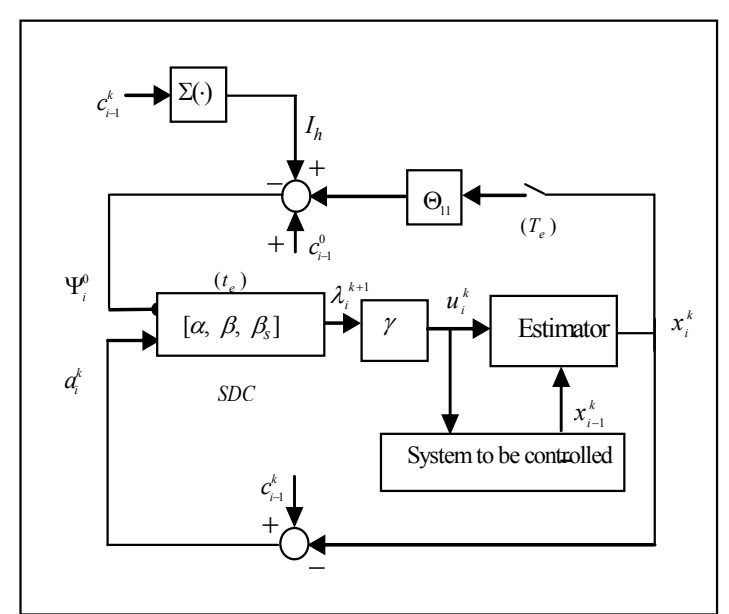

Figure 5. Optimal SDC with delayed state information

\section{Sampled Tracking with Delayed Output Feedback}

Consider now the general sampled tracking problem when delayed plant output measurements $y_{i-1}^{k}$ are only available. This problem can be solved incorporating an observer in the SCD developed in Section 3 in order to estimate the delayed plant state $x_{i-1}^{k}$ based on $y_{i-1}^{k}$.

The $x_{i-1}^{k}$ estimation can be realized by using a discrete reduced-order Luenberger observer [12] with inputs $y_{i-1}^{k}$ and $u_{i}^{k}$. Rewrite the equations (6) of the system to be controlled as

$x_{\mathrm{i}}^{k+1}=\left[\begin{array}{ll}f_{11} & f_{12} \\ f_{21} & f_{21}\end{array}\right]\left[\begin{array}{l}x_{1, i}^{\mathrm{k}} \\ x_{2, \mathrm{i}}^{\mathrm{k}}\end{array}\right]+\left[\begin{array}{l}h_{1} \\ h_{2}\end{array}\right] u_{\mathrm{i}}^{\mathrm{k}}$

$y_{i-1}^{k}=C x_{\mathrm{i}-1}^{\mathrm{k}}=x_{1, \mathrm{i}-1}^{\mathrm{k}}$

where $x_{1, \mathrm{i}}^{\mathrm{k}} \in \mathfrak{R}^{m}$ and $x_{2, \mathrm{i}}^{\mathrm{k}} \in \mathfrak{R}^{n-m}$. The vector $x_{2, i-1}^{k}$ can be estimated by the reducedorder Luenberger observer

$$
\begin{aligned}
& z_{\mathrm{i}}^{\mathrm{k}+1}=F z_{\mathrm{i}}^{\mathrm{k}}+G x_{1, i}^{k}+H u_{\mathrm{i}}^{\mathrm{k}} \\
& z_{\mathrm{i}}^{\mathrm{k}}=\hat{x}_{2, \mathrm{i}}^{\mathrm{k}}-L x_{1, i}^{k} \\
& \hat{x}_{2, \mathrm{i}}^{\mathrm{k}}=z_{\mathrm{i}}^{\mathrm{k}}+L x_{1, i}^{k}
\end{aligned}
$$

where

$$
\begin{aligned}
& F=\left(f_{22}-L f_{12}\right) \\
& G=\left(f_{22}-L f_{12}\right) L+\left(f_{21}-L f_{11}\right) \\
& H=\left(f_{22}-L f_{12}\right)
\end{aligned}
$$


and the matrix $L \in \mathfrak{R}^{(n-m) \times m}$ is chosen in (29a) so that the matrix $F$ to have zero spectrum. Thus we obtain the estimate

$$
\hat{x}_{i-1}^{k}=\left[\begin{array}{ll}
x_{1, i-1}^{k} & \hat{x}_{2, i-1}^{k}
\end{array}\right]^{T} .
$$

Replacing $x_{i-1}^{k}$ by $\hat{x}_{i-1}^{k}$ in (24) we can realize an output trajectory tracking with two switching period of delay:

$$
y_{i+2}^{0}=C c_{i}^{0}, \forall i=0,1,2, \ldots .
$$

For simplified SDC with delayed plant output information, the controller parameters are determined as

$\beta^{d}=M^{-1}, \psi_{i}^{0}=\left[c_{i}^{0}-A_{d}^{q} \hat{x}_{i}^{0}\right]$.

We can also replace $x_{i}^{k}$ by $\hat{x}_{i}^{k}$ in (15) and (19) obtaining thus an optimal SDC with delayed plant output information, which parameters are

$$
\begin{aligned}
& \alpha=A_{d}^{-T} \\
& \beta=A_{d}^{-T} Q \\
& \gamma=R^{-1} A_{d}^{-T} \\
& \beta_{i}^{d}=\Theta_{12}^{-1} \\
& a_{i}^{k}=c_{i-1}^{k}-\hat{x}_{i}^{k} \\
& \psi_{i+1}^{0}=c_{i}^{0}-\Theta_{11} \hat{x}_{i}^{0}-I_{h} .
\end{aligned}
$$

The corresponding controller structure is illustrated in Figure 6.

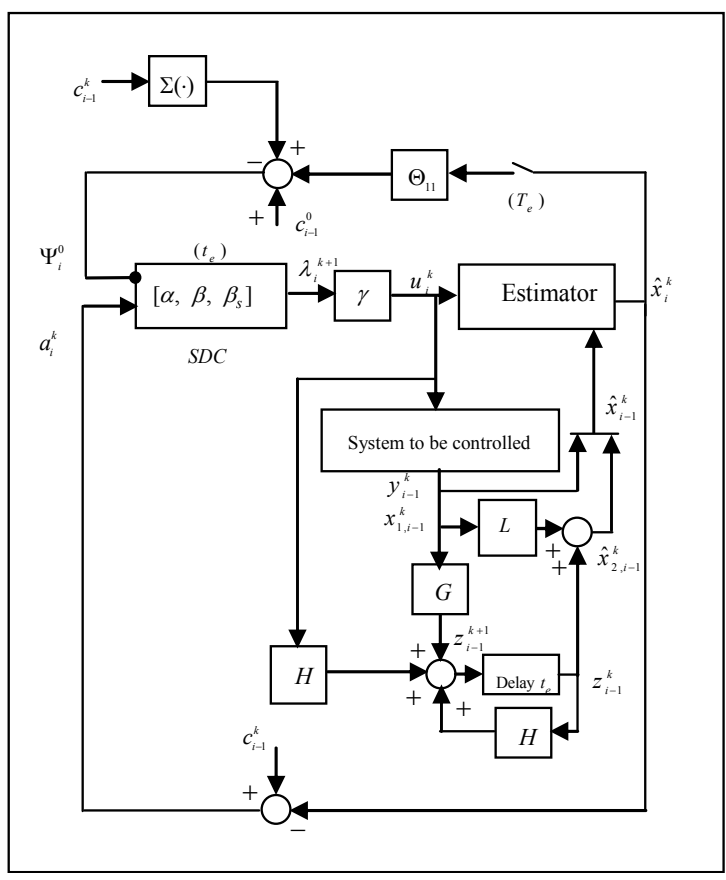

Figure 6. Optimal SDC with delayed plant output information

\section{Numerical Simulations}

The performances of the proposed SDC have been analysed by numerical simulation for the second order plant model

$\dot{x}(t)=A x(t)+B u(t)$

$y=C x(t)$

with $A=\left[\begin{array}{cc}0 & 1 \\ 2 & -1\end{array}\right], B=\left[\begin{array}{l}0 \\ 2\end{array}\right], C=\left[\begin{array}{ll}1 & 0\end{array}\right]$, and delayed output measurements

$y^{*}\left(t-T_{e}\right)=y^{*}\left(t-q \cdot t_{e}\right)$.

The corresponding two-time-scale sampleddata system is

$x_{i}^{k+1}=A_{d} x_{i}^{k}+B_{d} u_{i}^{k}, k=0,1, \ldots, q-1$

$y_{i-1}^{k}=C x_{i-1}^{k}$.

The switching period $T_{e}=q t_{e}=1 \mathrm{~s}$ and $q=2,4,20 ; \quad t_{e}=0.5,0.25,0.05$ have been chosen corresponding to following sampleddata system matrices:

1) $q=2, t_{e}=0.5$

$A_{d}=\left[\begin{array}{ll}1.222 & 0.427 \\ 0.854 & 0.795\end{array}\right] \quad B_{d}=\left[\begin{array}{l}0.222 \\ 0.854\end{array}\right] ;$

2) $q=4, t_{e}=0.25$

$A_{d}=\left[\begin{array}{ll}1.058 & 0.226 \\ 0.452 & 0.832\end{array}\right] \quad B_{d}=\left[\begin{array}{l}0.058 \\ 0.452\end{array}\right] ;$

3) $q=20, t_{e}=0.05$

$A_{d}=\left[\begin{array}{ll}1.003 & 0.049 \\ 0.098 & 0.954\end{array}\right] \quad B_{d}=\left[\begin{array}{l}0.003 \\ 0.098\end{array}\right]$.

The desired state trajectories has been defined as

$c(t)=\left[\begin{array}{l}c_{1}(t) \\ c_{2}(t)\end{array}\right]=\left[\begin{array}{c}-0.17 t^{3}+2.5 t^{2}-10 t+10 \\ -0.51 t^{2}+5^{*} t-10\end{array}\right]$.

First, sampled tracking with delayed state measurements has been simulated for simplified and optimal SDC. The simplified SDC parameters were determined from $\alpha=\exp \left(\delta t_{e}\right), \delta=\left[\begin{array}{cc}-2 & 8 \\ -7 & 10\end{array}\right]$ and $\gamma=\left[\begin{array}{ll}10 & 10\end{array}\right]$.

The results obtained are given in Figure 7. 

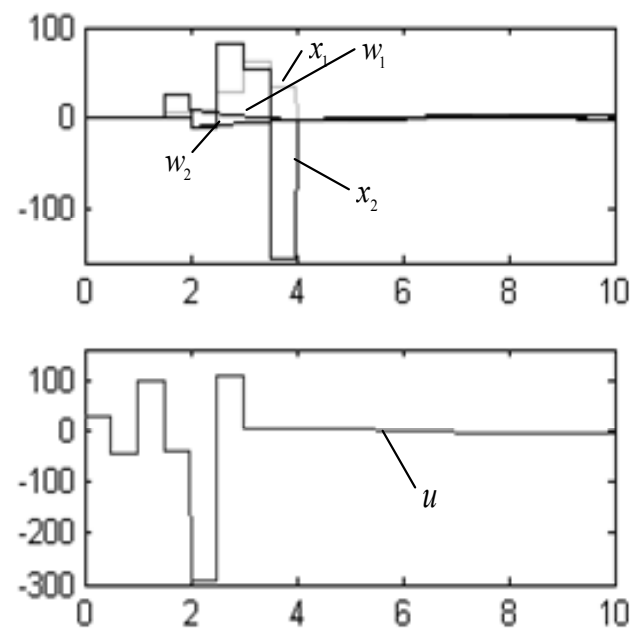

(a) $q=2, t_{e}=0.5 \mathrm{~s}, T_{e}=1 \mathrm{~s}$.
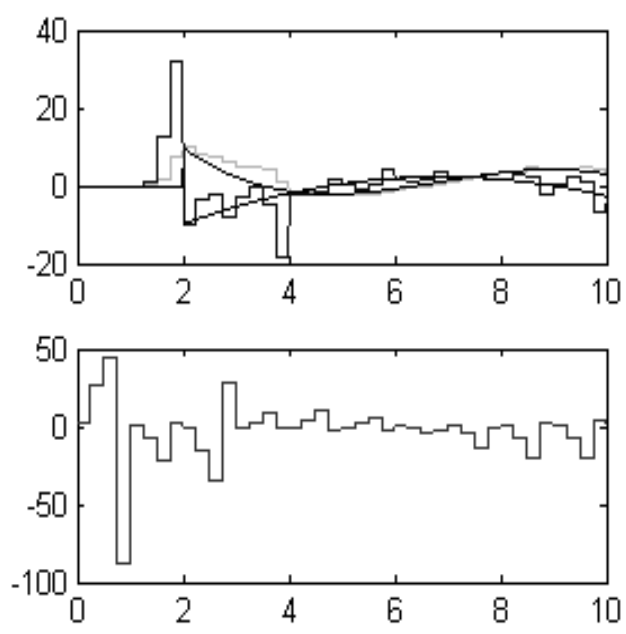

(b) $q=4, t_{e}=0.25 \mathrm{~s}, T_{e}=1 \mathrm{~s}$.
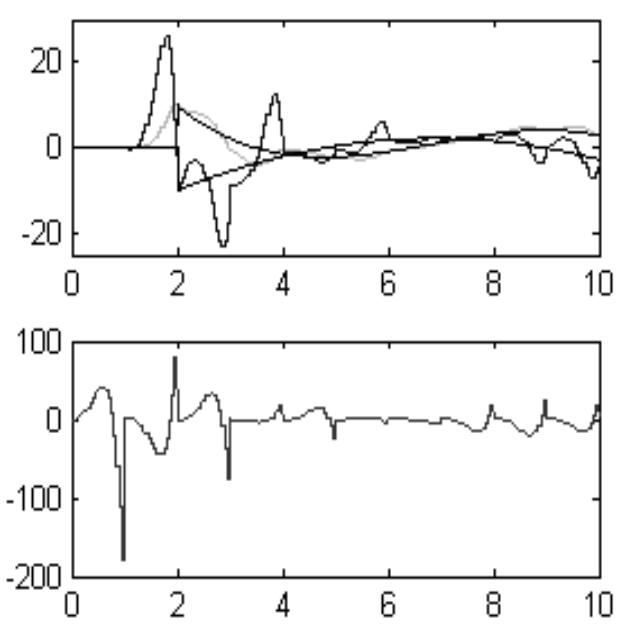

(c) $q=20, t_{e}=0.05 \mathrm{~s}, T_{e}=1 \mathrm{~s}$.

Figure 7. Optimal SDC with delayed state information
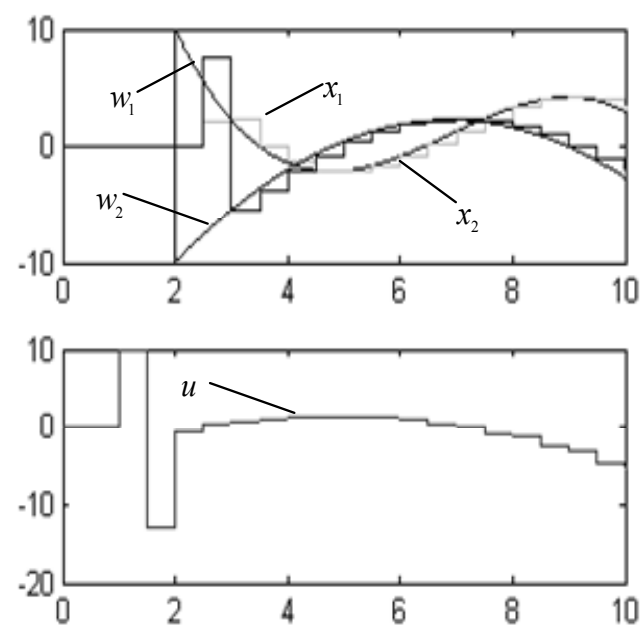

(a) $q=2, t_{e}=0.5 \mathrm{~s}, T_{e}=1 \mathrm{~s}$.
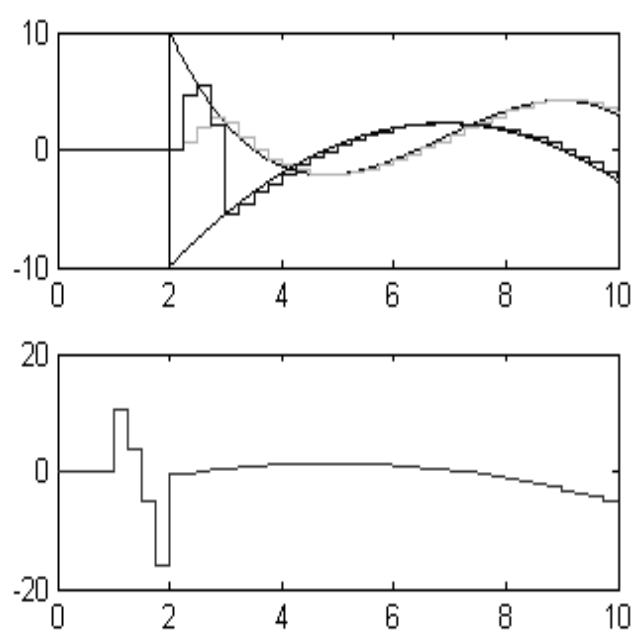

(b) $q=4, t_{e}=0.25 \mathrm{~s}, T_{e}=1 \mathrm{~s}$.
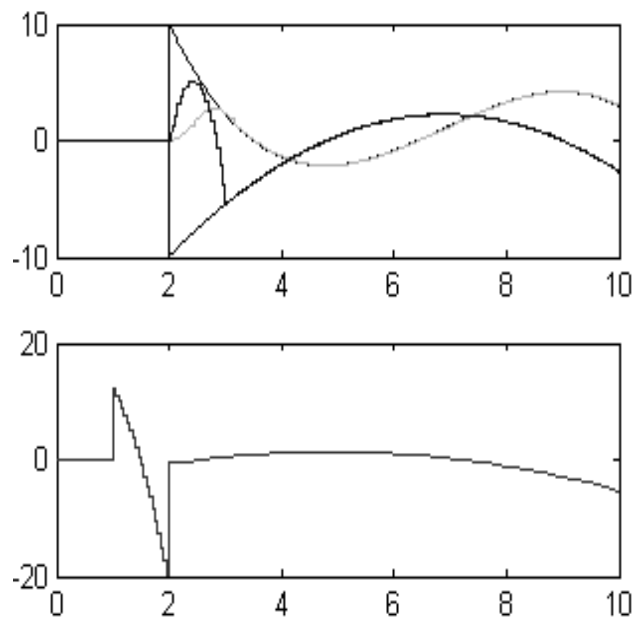

(c) $q=20, t_{e}=0.05 \mathrm{~s}, T_{e}=1 \mathrm{~s}$.

Figure 8. Optimal SDC with delayed plant state information 
Note that to facilitate the tracking performance evaluation, in Figures $7-10$ the trajectory

$$
w(t)=\left[\begin{array}{l}
w_{1}(t) \\
w_{2}(t)
\end{array}\right]=\left[\begin{array}{l}
c_{1}\left(t-2 T_{e}\right) \\
c_{2}\left(t-2 T_{e}\right)
\end{array}\right]
$$

is presented instead of $c(t)$.

For the optimal SDC with delayed state measurements, $Q=0.01 I_{2}$ and $R=100$ have been chosen and the controller parameters have been determined by (17)-(23). The corresponding simulation results are given in Figure 8.

Then sampled tracking with delayed plant output measurements has been simulated for the optimal SDC. The controller parameters have been determined from (30) and (29) for $Q=0.01 I_{2}$ and $R=100$. The simulation results are presented in Figure 9.

From Figure 7 and Figure 8 it can be seen that the delayed plant state $x_{i-1}^{k}$ follows perfectly the desired state trajectory in switching instants with two switching periods of delay. In turn, Figure 9 shows that the delayed plant output $y_{i-1}^{k}$ strictly follows the desired output trajectory $c_{1}(t)$ with two switching periods of delay. In the trajectory tracking using simplified SDC there exist oscillations between two switching instants except in the case where $q$ is equal to the order of the system. In the trajectory tracking by optimal SDC both for delayed state and output measurements, these oscillations are significantly reduced. Figure 9 shows also that the observer state $\hat{x}_{2, i-1}^{k}$ tracks well the desired state trajectory $c_{2}(t)$ with two switching periods of delay.

Finally, to analyse the robustness of the optimal SDC with delayed output information, the parameter variations in the plant model have been realized as

$$
A=\left[\begin{array}{cc}
0 & 1 \\
2+0.1 \sin (5 t) & -1+0.2 \sin (10 t)
\end{array}\right]
$$

and

$$
B=\left[\begin{array}{c}
0 \\
2+0.1 \sin (5 t)
\end{array}\right] .
$$
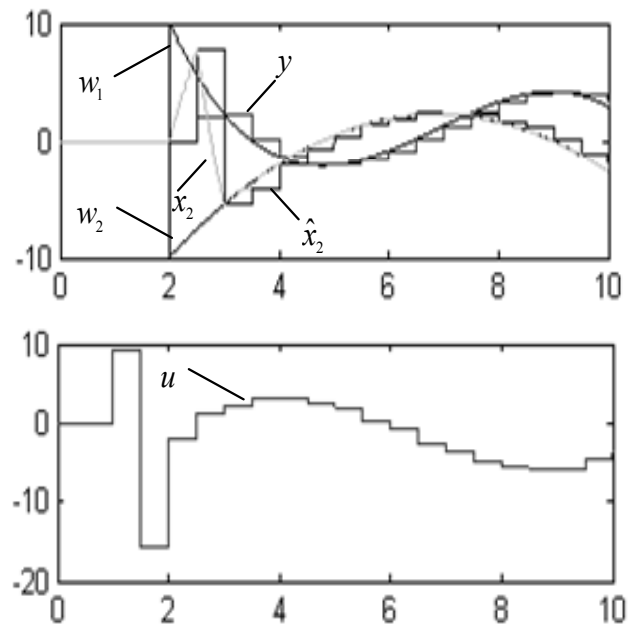

(a) $q=2, t_{e}=0.5 \mathrm{~s}, T_{e}=1 \mathrm{~s}$.
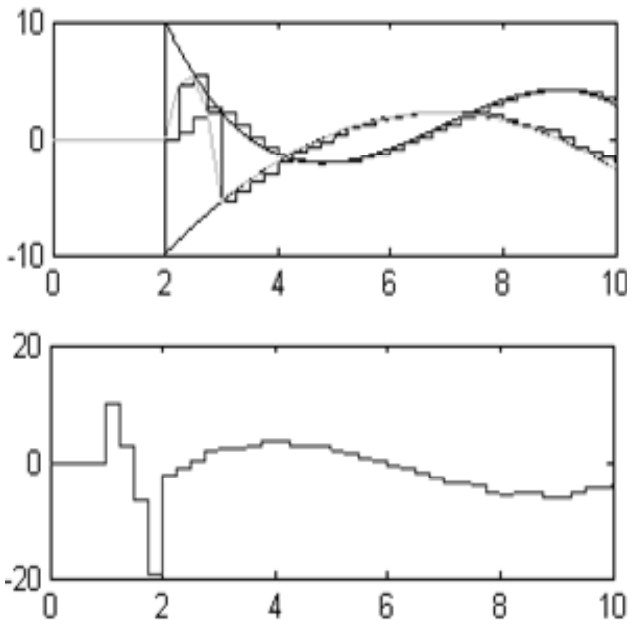

(b) $q=4, t_{e}=0.25 \mathrm{~s}, T_{e}=1 \mathrm{~s}$.
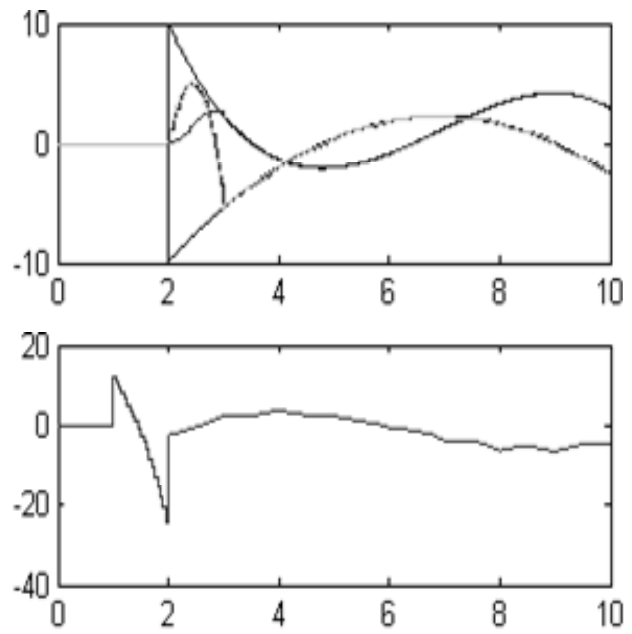

(c) $q=20, t_{e}=0.05 \mathrm{~s}, T_{e}=1 \mathrm{~s}$.

Figure 9. Optimal SDC with delayed 

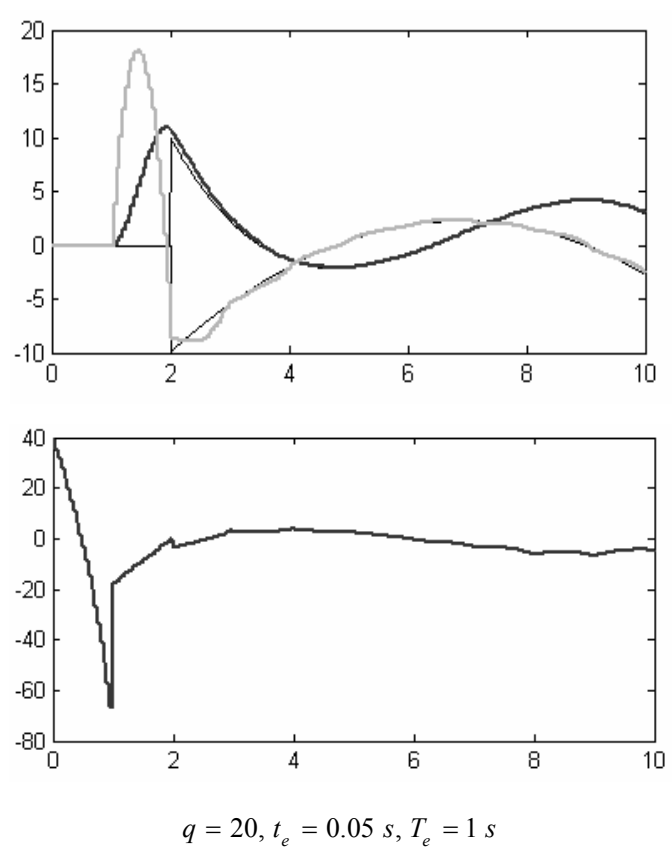

Figure 10. Optimal SDC with delayed output information in the case of system parameter variations

The corresponding trajectory tracking results, given in Figure 10, show the robustness and the efficiency of the proposed SDC.

\section{Conclusions}

In this paper, simplified and optimal twotime-scale sampled-data controllers are proposed for trajectory tracking control of linear plants with delayed state or output measurements. These controllers can achieve perfect trajectory tracking in switching instants with two switching periods of delay and are sufficiently robust against variations of the plant parameters. The proposed optimal controllers enable also to minimize the oscillations between switching instants.

\section{REFERENCES}

1. LENNARTSON, B., Multirate Sampleddata Control of Two-Time-Scale Systems, IEEE Trans. on Automatic Control, Vol. 34(6), 1989, pp. 642-644.

2. OLOOMI, H. M., P. I.-H. LIN, Two-timeScale Digital Controller Design for a Position Servo system, Proc. IEEE/IAS Conf. on Industrial Automation and Control: Emerging Tech., 1995, pp. 663-668.

3. KONCAR, V., C. VASSEUR, Piecewise Functioning Systems: Bi-sampled
Controllers, Studies in Informatics and Control, Vol. 11(2), 2002.

4. HAN, D. K., P. H. CHANG, A Robust Two-Time-Scale Control Design for a Pneumatic Vibration Isolator, Proc. 46th IEEE Conf. Decision and Control, New Orleans, 2007, pp. 1666-1672.

5. BECERRIL-ARREOLA, R., A. G. AGHDAM, V. D. YURKEVICH, Decentralised Two-time-scale Motions Control Based on Generalised Sampling, IET Control Theory \& Applications, Vol. 1(5) , 2007, pp. 1477-1486.

6. KABAMBA, P. T., Control of Linear Systems Using Generalized SampledData Hold Functions, IEEE Trans. On Automatic Control, Vol. AC-32(9), 1987, pp. 772-783.

7. CHAMROO, A., I. SIMEONOV, C. VASSEUR, N. CHRISTOV, On the Piecewise Continuous Control of Methane Fermentation Processes, Studies in Informatics and Control, Vol. 17(2), 2008, pp. 181-188.

8. WANG, H.P, C. VASSEUR C., V. KONCAR, A. CHAMROO, N. CHRISTOV, Design and Implementation of Robust Hybrid Control of Vision Based Underactuated Mechanical Nonminimum Phase Systems, Studies in Informatics and Control, Vol. 19(1), 2010, pp. 35-44.

9. TITTUS, M., B. EGART, Control Design for Integrator Hybrid Systems, IEEE Trans. on Automatic Control, Vol.43, No.4, 1998, pp. 491-500.

10. BRANICKY, M. S., V. S. BORKAR, S. K. MITTER, A Unified Framework for Hybrid Control: Model and Optimal Control Theory, IEEE Trans. on Automatic Control, vol. 43(1), 1998, pp. 31-45.

11. URIKURA, S., A. NAGATA, RippleFree Deadbeat Control for SampledData Systems, IEEE Trans. on Automatic Control, Vol. AC-32(6), 1987, pp. 474-482.

12. LUENBERGER, D. G., An Introduction to Observers, IEEE Trans. on Automatic Control, Vol. 16(6), 1971, pp. 596-602. 